TITLE:

\title{
Photothermally controlled Marangoni flow around a micro bubble
}

$\operatorname{AUTHOR}(S)$ :

Namura, Kyoko; Nakajima, Kaoru; Kimura, Kenji; Suzuki, Motofumi

\section{CITATION:}

Namura, Kyoko ...[et al]. Photothermally controlled Marangoni flow around a micro bubble. Applied Physics Letters 2015, $106(4): 043101$.

ISSUE DATE:

2015-01-26

URL:

http://hdl.handle.net/2433/193466

\section{RIGHT:}

Copyright 2015 American Institute of Physics. This article may be downloaded for personal use only. Any other use requires prior permission of the author and the American Institute of Physics 


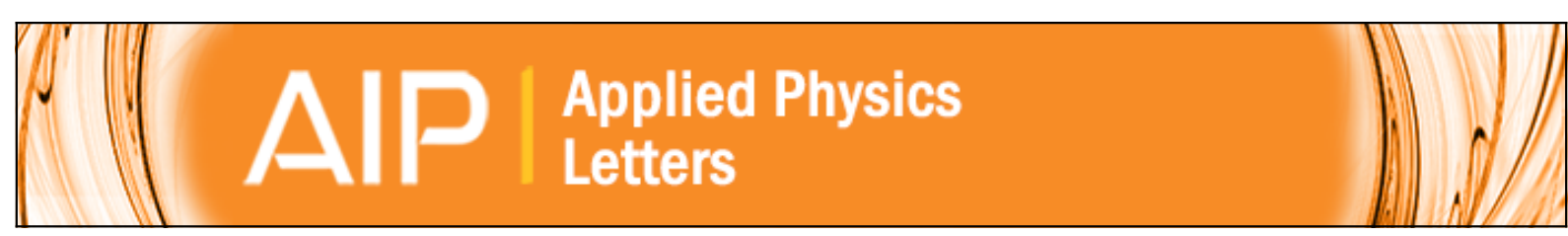

\section{Photothermally controlled Marangoni flow around a micro bubble}

Kyoko Namura, Kaoru Nakajima, Kenji Kimura, and Motofumi Suzuki

Citation: Applied Physics Letters 106, 043101 (2015); doi: 10.1063/1.4906929

View online: http://dx.doi.org/10.1063/1.4906929

View Table of Contents: http://scitation.aip.org/content/aip/journal/apl/106/4?ver=pdfcov

Published by the AIP Publishing

\section{Articles you may be interested in}

Thin-liquid-film flow on a topographically patterned rotating cylinder

Phys. Fluids 26, 042102 (2014); 10.1063/1.4869208

Simulation of micro gas bubble generation of uniform diameter in an ultrasonic field by a boundary element method

Phys. Fluids 18, 108102 (2006); 10.1063/1.2364139

On the paradox of thermocapillary flow about a stationary bubble

Phys. Fluids 18, 072101 (2006); 10.1063/1.2218767

Buoyancy and surface tension driven convection around a bubble

Phys. Fluids 18, 057104 (2006); 10.1063/1.2202931

Numerical investigation of thermocapillary flow around a bubble

Phys. Fluids 11, 18 (1999); 10.1063/1.869900

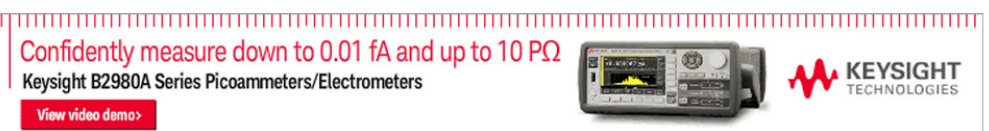




\title{
Photothermally controlled Marangoni flow around a micro bubble
}

\author{
Kyoko Namura, ${ }^{\text {a) }}$ Kaoru Nakajima, Kenji Kimura, and Motofumi Suzuki \\ Department of Micro Engineering, Kyoto University, Kyoto daigaku-Katsura, Kyoto 615-8540, Japan
}

(Received 10 December 2014; accepted 18 January 2015; published online 26 January 2015)

\begin{abstract}
We have experimentally investigated the control of Marangoni flow around a micro bubble using photothermal conversion. Using a focused laser spot acting as a highly localized heat source on $\mathrm{Au}$ nanoparticles/dielectric/Ag mirror thin film enables us to create a micro bubble and to control the temperature gradient around the bubble at a micrometer scale. When we irradiate the laser next to the bubble, a strong main flow towards the bubble and two symmetric rotation flows on either side of it develop. The shape of this rotation flow shows a significant transformation depending on the relative position of the bubble and the laser spot. Using this controllable rotation flow, we have demonstrated sorting of the polystyrene spheres with diameters of $2 \mu \mathrm{m}$ and $0.75 \mu \mathrm{m}$ according to their size. ( 2015 AIP Publishing LLC. [http://dx.doi.org/10.1063/1.4906929]
\end{abstract}

Temperature gradient along a gas-liquid interface causes surface tension differential along the surface, which results in a liquid motion. This phenomenon has long been known as the Marangoni or thermocapillary effect. ${ }^{1,2}$ Fluid manipulation mediated by this Marangoni effect has been investigated numerically ${ }^{3-6}$ and experimentally ${ }^{7-13}$ and its potential for microfluidic control has been shown. Especially, a small bubble of the order of $10 \mu \mathrm{m}$ was shown to be capable of developing a jet like Marangoni flow with a high velocity of more than $100 \mathrm{~mm} / \mathrm{s}$ using a thin wire heater of $0.1 \mathrm{~mm}$ diameter. ${ }^{10}$ If we can manipulate this strong flow in a micro channel, it could be a powerful driving force in microfluidics. A key issue in manipulating the Marangoni flow around a micro bubble is the difficulty of controlling the temperature gradient at a micrometer scale flexibly and precisely.

We have recently demonstrated the self-assembly of $\mathrm{Au}$ nanoparticles $/ \mathrm{SiO}_{2}$ nanocolumns $/ \mathrm{SiO}_{2} / \mathrm{Ag}$ mirror multilayered structure, i.e., the local plasmon resonator ${ }^{14-17}$ using a dynamic oblique deposition (DOD) technique ${ }^{18-21}$ and have shown its capability as a themoplasmonic ${ }^{22}$ heater. A remarkable feature of the local plasmon resonators is strong and highly localized optical absorption in the Au nanoparticles layer $(\sim 97 \%){ }^{16}$ This feature allows them to convert almost all of the incident light to heat within the thin surface layer $(\sim 10 \mathrm{~nm})$. Taking advantage of their localized photothermal conversion, we demonstrate that their strong photoacoustic emission is eight times larger than that of a graphite which has a high optical absorption of $85 \% .{ }^{16}$ Another feature that makes the local plasmon resonators attractive as a heater is the existence of a layer of $\mathrm{SiO}_{2}$ nanocolumns under the Au nanoparticles. In our previous work, the photoacoustic emission was enhanced by a factor of three by inserting a 340-nm-thick porous $\mathrm{SiO}_{2}$ nanocolumn layer whose thermal conductivity was about 3-4 times lower than that of a plane $\mathrm{SiO}_{2}$ layer. This result suggests that the $\mathrm{SiO}_{2}$ nanocolumn layer reduces in-plane and out-of-plane heat transfer from Au nanoparticles to the substrate. Accordingly, the generated heat is highly localized under the spot of the irradiated light.

\footnotetext{
${ }^{a}$ Electronic mail: namura.kyoko.57r@st.kyoto-u.ac.jp; Research Fellow of the Japan Society for the Promotion of Science.
}

In other words, a focused light on the local plasmon resonator can be used as a localized and mobile point-heat-source at the micrometer scale.

Some researchers have recently demonstrated using photothermal conversion to control the Marangoni flow around micro bubbles. ${ }^{23-29}$ However, there are only a few studies that have observed the detailed dependence of the Marangoni flow on the relative position of the bubble and the laser spot, i.e., the heat source. In this study, we demonstrate drastic transformation of the Marangoni flow around a micro bubble by controlling the heating position at a micrometer scale using a well-localized photothermal conversion of the local plasmon resonator. In addition, we propose a size-based particle sorting method as an application of the controlled Marangoni flow around a micro bubble.

We prepared the local plasmon resonators with $\mathrm{Au}$ nanoparticles $/ \mathrm{SiO}_{2}$ nanocolumns/ $/ \mathrm{SiO}_{2} / \mathrm{Ag}$ structures by using DOD. Their preparation method is essentially the same as that reported in prior publications. ${ }^{14-16}$ First, a smooth $\mathrm{Ag}$ layer $(200 \mathrm{~nm})$ and $\mathrm{a} \mathrm{SiO}_{2}$ layer $(60 \mathrm{~nm})$ were deposited on a glass substrate. On the $\mathrm{SiO}_{2}$ layer, a $300 \mathrm{~nm}$ thick $\mathrm{SiO}_{2}$ nanocolumn layer was prepared. During the deposition of the nanocolumns, the deposition angle measured from the surface normal was fixed at $80^{\circ}$, and the substrate was continuously and rapidly rotated. An Au nanoparticle layer $(\sim 8.5 \mathrm{~nm})$ was then deposited at a deposition angle of $73.4^{\circ}$ on the nanocolumns. The optical reflection of the sample in water was measured at an incidence angle of $2^{\circ}$ using a single-beam spectrophotometer. Finally, we obtained local plasmon resonators with high optical absorption of $89 \%$ in water at the wavelength of $785 \mathrm{~nm}$, at which we performed photothermal conversion.

Figure 1 shows a schematic of experimental setup for microfluidic control. A shallow cell, of $50-\mu \mathrm{m}$ height, was created on the local plasmon resonator and filled with water in which polystyrene (PS) spheres (R0200, Thermo Scientific) with a diameter of $2 \mu \mathrm{m}$ were dispersed. Although these PS spheres are necessary to visualize the fluid motion, their water suspension often contains a surfactant that considerably weakens the surface tension. Therefore, we carefully chose a water suspension of PS spheres containing only 


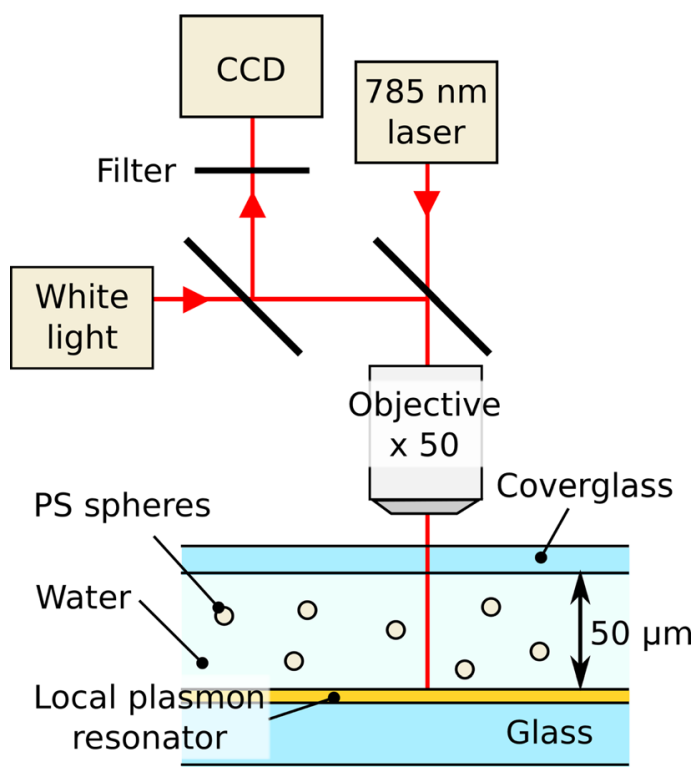

FIG. 1. Schematic of the experimental setup used in the study.

a small amount of surfactant and diluted it with distilled water to a ratio of 1:20 (particle number density: $\sim 1$ $\times 10^{8} \mathrm{~cm}^{-3}$ ). The prepared sample cell was observed using an objective lens $(50 \times, \mathrm{NA}=0.42)$ in an upright microscope (M-scope, Synos) under white light illumination. The objective lens was also utilized to focus the laser for photothermal conversion on the local plasmon resonator. The diameter of the laser spot was less than $10 \mu \mathrm{m}$, and the laser power at the sample surface was tunable from $0 \mathrm{~mW}$ to $13.5 \mathrm{~mW}$. Fluid motion visualized by the motion of PS spheres was recorded by a CCD (DMK 41AU02, The Imaging Source), whose exposure time was set at $5 \mathrm{~ms}$. A short-pass filter was placed in front of the CCD to eliminate the 785-nm laser source.

With the laser at the maximum power of $13.5 \mathrm{~mW}$ focused on the local plasmon resonator, a micro bubble with a diameter of 40-50 $\mu \mathrm{m}$ was generated because of the highly localized photothermal conversion. The laser power was tuned down to $12 \mathrm{~mW}$ and the irradiated spot moved to a position near the created bubble. When the laser position was set next to the bubble, a unique and rapid flow developed around the bubble. Figure 2(a) shows an optical image of the typical flow around the bubble, whose motion is described in Fig. 2(b). The flow pattern basically consists of a strong main flow towards the bubble and two symmetric rotation flows on either side of it. The velocity of the flow is at least of the order of $\mathrm{mm} / \mathrm{s}$ since the images of the PS spheres are elongated in the direction of the flow. We assert that this rapid flow is driven by the Marangoni effect because of the following reasons. Due to the localized photothermal conversion of the local plasmon resonator, a portion of the bubble surface near the laser spot is heated up. The surface tension of the heated point is significantly weakened and generates a strong shear force along the bubble surface that drags the surrounding fluid and induces rapid flow. Here, we provide a rough estimation of this Marangoni effect. When the temperature varies along the bubble surface from $T_{h}$ at the hot pole near the laser spot to $T_{c}$ at the cold pole, the average shear force on the bubble surface is given by $\bar{\tau}=\left(\sigma\left(T_{c}\right)-\sigma\left(T_{h}\right)\right) / \pi r$. In this equation, $\sigma(T)$ is the surface tension of water at temperature $T$ and $r$ is the radius of the bubble. If the fluid was simply a Newtonian fluid with constant viscosity, $\mu$, the balance of the viscous stress and the shear force is given by $\bar{\tau}=\mu \nabla v$, where $\nabla v$ is the gradient of the flow velocity around the bubble. Assuming that the flow velocity is zero on the cell wall, we have $\nabla v \simeq v_{p} / h$, where $v_{p}$ is velocity of the observed PS spheres and $h$ is the distance between those PS spheres and the cell wall. In our experimental conditions, $h \simeq 2 \mu \mathrm{m}$ because the observed PS spheres were distributed $\sim 2 \mu \mathrm{m}$ above the bottom surface of the cell considering the depth of focus. In addition, $r \simeq 30 \mu \mathrm{m}$ and $v_{p}$ was at least $10-20 \mathrm{~mm} / \mathrm{s}$ near the bubble surface. The values of $\mu$ and $\sigma(T)$ of water at around room temperature can be found in the literature. ${ }^{30,31}$ By using the equations above, the temperature gradient along the bubble surface is estimated to be $\nabla T=T_{h}-T_{c} \simeq 5 \mathrm{~K}$ around room temperature. This result suggests that it is reasonable to classify the observed rapid flow as Marangoni flow. A more precise analysis would require the determination of the temperature distribution around the bubble at a micrometer scale. Instead, we investigated qualitative changes in the developed flow around the bubble by systematically varying the relative position of the laser spot and the bubble because the Marangoni effect is known to be very sensitive to the temperature gradient.

Figure 3 shows the transformation of the flow around the micro bubble at different positions of the laser spot. Please note that all these images were obtained by a sequence of observations on the same bubble. When the laser spot was located at or near the center of the bubble, there was no distinct flow (Fig. 3(a)). However, when the laser position was moved slightly away from the center (Fig. 3(b)), a rapid flow was suddenly observed to develop. This flow also consists of a strong main flow towards the bubble and two symmetric rotation flows with a distinct appearance

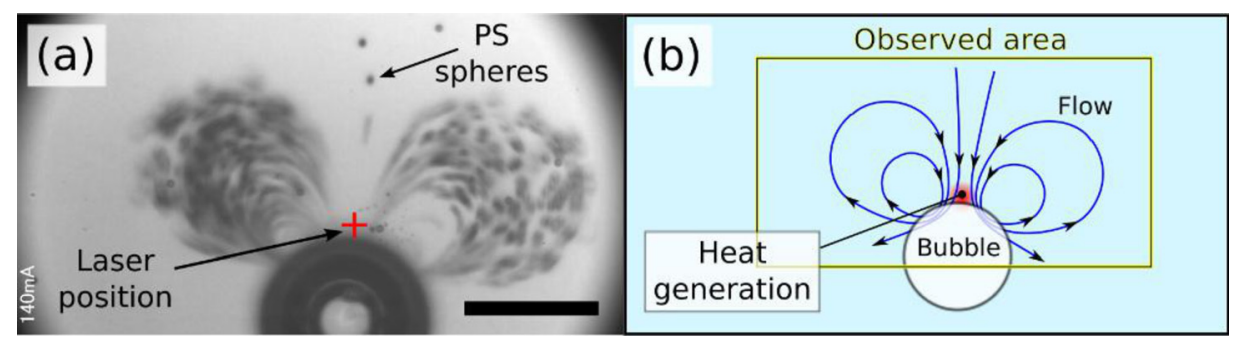

FIG. 2. (a) Microscopic image of the rapid flow around the micro bubble induced by the photothermal conversion of the local plasmon resonator. The red crosses show the laser position, the small black dots are the PS spheres, and the big black circle is the micro bubble with a diameter of $\sim 65 \mu$ m, respectively. Scale bar: $50 \mu \mathrm{m}$. (b) Sketch of the flow around the bubble. (Multimedia view) [URL: http://dx.doi.org/10.1063/1.4906929.1] 


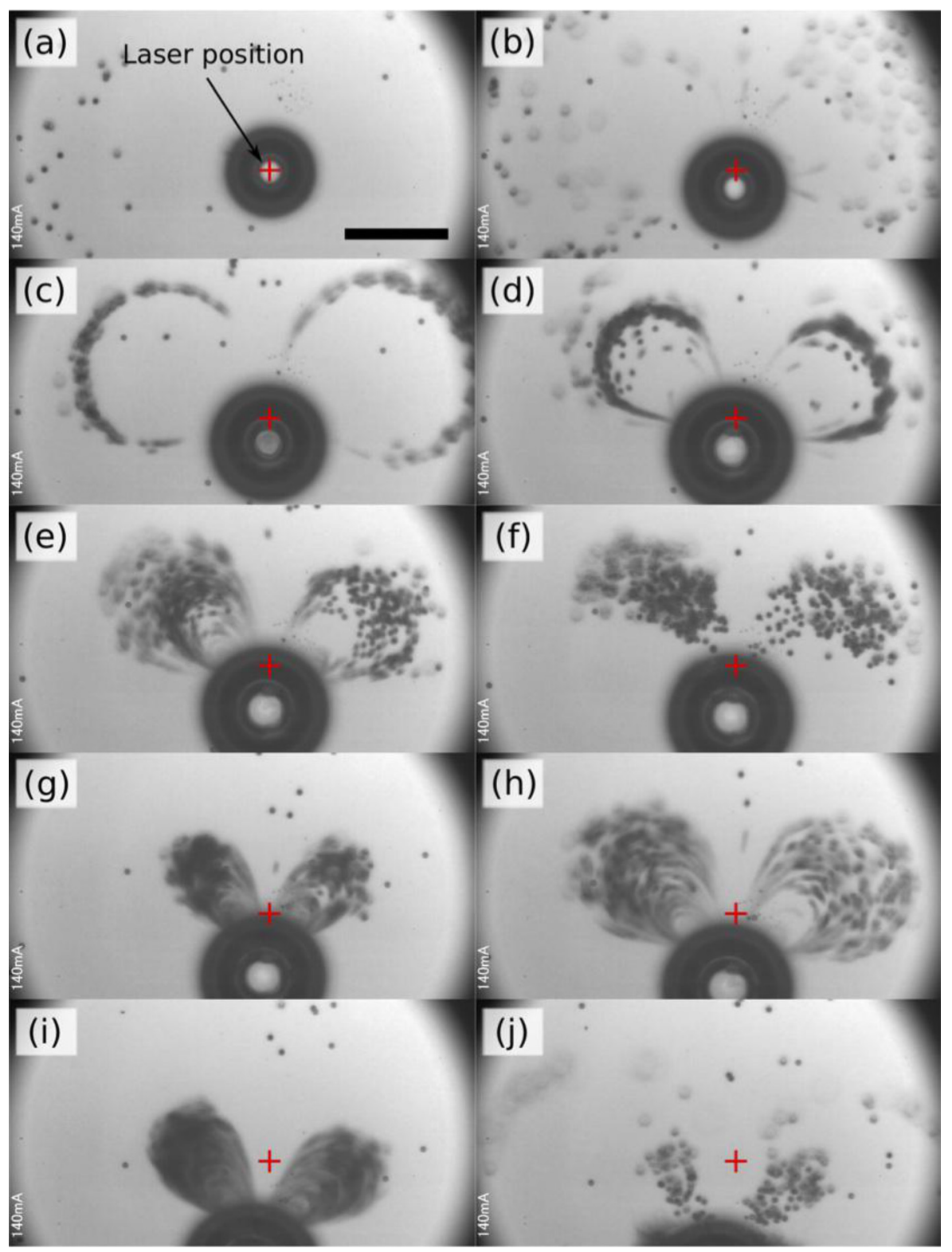

FIG. 3. (a)-(j) Series of microscopic images showing dependence of the transformation of the flow around the micro bubble on the position of the laser spot. The red crosses show laser positions, the small black dots are PS spheres, and the big black circles are the micro bubble, respectively. Scale bar: $50 \mu \mathrm{m}$.

change from that of Fig. 2(a). In Fig. 3(a), the rotation flow drives and mixes the PS spheres rather than trapping them. As the laser position is gradually moved away from the bubble center (Figs. 3(c)-3(e)), the shape of the rotation flow undergoes a significant transformation. The distinguishing characteristic of the rotation flow was observed at the laser position of Figs. 3(c) and 3(d), where the PS spheres are tidily ranged in two symmetric circular shapes. The radius of the circle was tunable by the laser position. Just before the laser moved out from the bubble (Fig. 3(f)), there was a region where the PS spheres could not be driven. When the laser spot was moved to the edge of the bubble (Fig. 3(g)), the rapid flow developed again and the PS spheres collected and concentrated near the bubble. As the distance of the laser
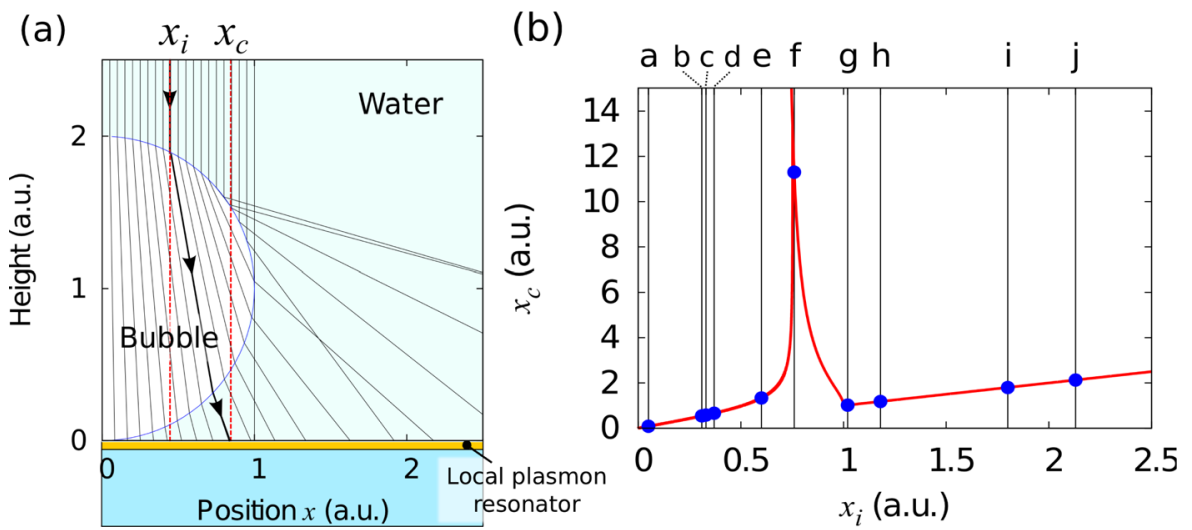

FIG. 4. (a) Cross-sectional view of the cell with the optical paths (black lines) indicated. The scale is normalized to the bubble radius. (b) Red line represents the photothermal conversion position, $x_{c}$, as a function of the laser irradiated position, $x_{i}$. Letters over the figure correspond to Figs. 3(a)-3(j) whose relative positions of the bubble and the laser spot are shown as blue dots. 


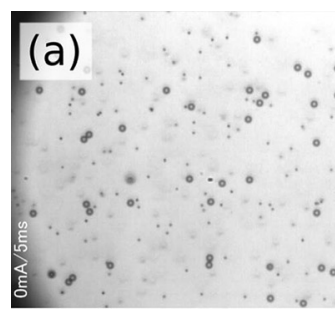

FIG. 5. (a) Microscopic image of the sample cell before the laser irradiation, in which PS spheres with diameters of $2 \mu \mathrm{m}$ and $0.75 \mu \mathrm{m}$ are well dispersed. (b) The controlled Marangoni flow around a photothermally created micro bubble. (c) Distribution of the PS spheres after the sorting by Marangoni flow. Scale bar: $50 \mu \mathrm{m}$.

spot from the bubble edge was increased, the shape of the rotation flow varied again (Figs. 3(g)-3(i)). Finally, when the distance of the laser spot from the bubble exceeded $\sim 28 \mu \mathrm{m}$, the rapid flow stopped. These results suggest that by tuning the relative position of the laser spot and the bubble, we can develop a rapid flow with a wide variety of functions such as driving, mixing, ranging, and collecting the PS spheres. For better understanding of the transformation of the flow, we focused our attention on light refraction and reflection at the bubble surface and evaluated the actual photothermal conversion position.

Figure 4(a) corresponds to a cross sectional view of the sample cell whose scale is normalized by the bubble radius. The black lines in Fig. 4(a) show ideal light paths going through a spherical vapor-water boundary. Although a ray of light splits into a number of rays after experiencing multiple refractions and/or reflections, we only consider the dominant light path for the photothermal heating of the bubble. Depending on the position of the laser irradiation, the position where the local plasmon resonator absorbs light varies significantly. Thus, we plot the photothermal conversion position, $x_{c}$, as a function of the laser irradiated position, $x_{i}$ (Fig. 4(b)). At around $x_{i}=0.75$, the value of $x_{c}$ has a distinct peak indicating that the photothermal conversion position is far away from the bubble due to total reflection although the laser spot is placed on the bubble.

The evaluated photothermal conversion positions for the observed flows in Fig. 3 are represented in Fig. 4(b). We normalized the laser position by the cross sectional bubble radius assuming that the contact angle of the sample cell wall with water is much lower than $90^{\circ}$. The laser position for Figs. 3(a)-3(j) is shown by vertical black lines in Fig. 4(b). Among them, position (f) shows the farthest photothermal conversion position from the bubble, followed by the position (j). Therefore, absence of the rapid flow in Figs. 3(f) and $3(\mathrm{j})$ is explained by their photothermal conversion position, which is too distant to present a significant temperature gradient around the bubble. Positions (e) and (h) are similar in the photothermal conversion position $\left(x_{c}=1.3\right.$ for (e) and $x_{c}=1.2$ for (h), respectively), whereby their rotation flows are equivalent in shape (Figs. 3(e) and 3(h)). There is a tendency for the PS spheres to concentrate near the bubble as the value of $x_{c}$ becomes larger, except for the position $(\mathrm{g})$. The high concentration of PS spheres observed in Fig. 3(g) may be due to the width of the actual laser spot, which results in a widespread value of $x_{c}$ from 1 to $\sim 2$ because of its rather exponential change around $x_{i}=1$ (Fig. 4(b)). Although the photothermal conversion position is shown to be right underneath the bubble for the laser position (a), no significant flow was observed in Fig. 3(a). This is because the water contact angle in the actual sample is not exactly zero and there is little water to drive at the center of the bubble. These results suggest that the flow development around the bubble can be characterized qualitatively by the position of the photothermal conversion. Further investigation of the transformation of the rotation flow requires numerical analysis that we plan to undertake in the future.

Here, we experimentally demonstrate the sorting of the PS spheres with different diameters as one of the applications of the photothermally controlled Marangoni flow around a micro bubble. We prepared and filled the sample cell with a 1:1 mixture of the water suspension of PS spheres with a diameter of $2 \mu \mathrm{m}$ (R0200, Thermo Scientific, diluted to $5 \%$ by water, particle number density: $\sim 1 \times 10^{8} \mathrm{~cm}^{-3}$ ) and of $0.75 \mu \mathrm{m}$ (42742, Alfa Aesar, diluted to $2 \%$ by water, particle number density: $\sim 2 \times 10^{9} \mathrm{~cm}^{-3}$ ). Fig. 5(a) shows the uniformly dispersed PS spheres with diameters of $2 \mu \mathrm{m}$ and $0.75 \mu \mathrm{m}$ before the laser irradiation. Following the experimental methods described above, we created a micro bubble and developed Marangoni flow around it using localized photothermal conversion of the local plasmon resonator. By optimizing the relative position of the laser spot and the micro bubble, the PS spheres with different diameters are trapped in the rotation flows with different radius (Fig. 5(b)). Figure 5(c) clearly shows that the PS spheres with diameters of $2 \mu \mathrm{m}$ and $0.75 \mu \mathrm{m}$ are segregate to different areas after the laser irradiation.

In conclusion, we have demonstrated drastic transformation of the Marangoni flow around a micro bubble by using highly localized photothermal conversion of the local plasmon resonator. The highly localized photothermal conversion of the local plasmon resonator allowed us to create a micro bubble in the sample cell and to control the temperature gradient around the bubble at a micrometer scale. As a result, a rapid flow towards the bubble and two rotation flows develop around the bubble and the shape of the rotation flow was proved to be controllable by the relative position of the bubble and the photothermal conversion position. Using photothermally controlled Marangoni flow, we demonstrated sorting of the PS spheres with diameters of $2 \mu \mathrm{m}$ and $0.75 \mu \mathrm{m}$ according to their size.

This work was supported by JSPS KAKENHI Grant Nos. 25286037 and 24.2362.

\footnotetext{
${ }^{1}$ A. V. Hershey, Phys. Rev. 56, 204 (1939).

${ }^{2}$ N. O. Young, J. S. Goldstein, and M. J. Block, J. Fluid Mech. 6, 350 (1959).

${ }^{3}$ K. Takahashi, J.-G. Weng, and C.-L. Tien, Microscale Thermophys. Eng. 3, 169 (1999).
} 
${ }^{4}$ S. M. O'Shaughnessy and A. J. Robinson, Microgravity Sci. Technol. 20, 319 (2008)

${ }^{5}$ S. M. O'Shaughnessy and A. J. Robinson, Heat Transfer Eng. 30, 1096 (2009).

${ }^{6}$ S. M. O'Shaughnessy and A. J. Robinson, Ann. N. Y. Acad. Sci. 1161, 304 (2009).

${ }^{7}$ B. A. Bezuglyi and N. A. Ivanova, Tech. Phys. Lett. 28, 828 (2002).

${ }^{8}$ N. A. Ivanova and B. A. Bezuglyi, J. Appl. Mechanics Tech. Phys. 46, 691 (2005).

${ }^{9}$ C. Buffone, K. Sefiane, and J. R. E. Christy, Phys. Fluids 17, 052104 (2005).

${ }^{10}$ H. Wang, X. F. Peng, D. M. Christopher, W. K. Lin, and C. Pan, International J. Heat Fluid Flow 26, 485 (2005).

${ }^{11}$ C. N. Baroud, M. R. de Saint Vincent, and J.-P. Delville, Lab Chip 7, 1029 (2007).

${ }^{12}$ A. S. Basu and Y. B. Gianchandani, J. Micromech. Microeng. 18, 115031 (2008).

${ }^{13}$ J.-P. Delville, M. R. de Saint Vincent, R. D. Schroll, H. Chraibi, B. Issenmann, R. Wunenburger, D. Lasseux, W. W. Zhang, and E. Brasselet, J. Opt. A: Pure Appl. Opt. 11, 034015 (2009).

${ }^{14}$ K. Namura, M. Suzuki, K. Nakajima, and K. Kimura, Opt. Lett. 36, 3533 (2011).

${ }^{15}$ K. Namura, M. Suzuki, K. Nakajima, and K. Kimura, Opt. Express 21, 8689 (2013).

${ }^{16}$ K. Namura, M. Suzuki, K. Nakajima, and K. Kimura, J. Appl. Phys. 114, 074308 (2013).
${ }^{17}$ K. Namura, K. Nakajima, K. Kimura, and M. Suzuki, J. Appl. Phys. 116, 024310 (2014).

${ }^{18}$ K. Robbie, M. J. Brett, and A. Lakhtakia, J. Vac. Sci. Technol., A 13, 2991 (1995)

${ }^{19}$ K. Robbie, L. J. Friedrich, S. K. Dew, T. Smy, and M. J. Brett, J. Vac. Sci. Technol., A 13, 1032 (1995).

${ }^{20}$ T. Karabacak, G.-C. Wang, and T.-M. Lu, J. Appl. Phys. 94, 7723 (2003).

${ }^{21}$ Y.-J. Jen and C.-F. Lin, Opt. Express 16, 5372 (2008).

${ }^{22}$ C.-S. Chiu, H.-Y. Chen, C.-F. Hsiao, M.-H. Lin, and S. Gwo, J. Phys. Chem. C 117, 2442 (2013).

${ }^{23}$ R. Muruganathan, Y. Zhang, and T. M. Fischer, J. Am. Chem. Soc. 128, 3474 (2006)

${ }^{24}$ A. T. Ohta, A. Jamshidi, J. K. Valley, H.-Y. Hsu, and M. C. Wu, Appl. Phys. Lett. 91, 074103 (2007).

${ }^{25}$ W. Hu, K. S. Ishii, and A. T. Ohta, Appl. Phys. Lett. 99, 094103 (2011)

${ }^{26}$ Y. Zheng, H. Liu, Y. Wang, C. Zhu, S. Wang, J. Cao, and S. Zhu, Lab Chip 11, 3816 (2011).

${ }^{27}$ S. Fujii, K. Kanaizuka, S. Toyabe, K. Kobayashi, E. Muneyuki, and M.-A. Haga, Langmuir 27, 8605 (2011).

${ }^{28}$ Y. Li, L. Xu, and B. Li, Appl. Phys. Lett. 101, 053118 (2012).

${ }^{29}$ C. Zhao, Y. Xie, Z. Mao, Y. Zhao, J. Rufo, S. Yang, F. Guo, J. D. Mai, and T. J. Huang, Lab Chip 14, 384 (2014).

${ }^{30}$ J. V. Sengers and J. T. R. Watson, J. Phys. Chem. Ref. Data 15, 1291 (1986).

${ }^{31}$ N. B. Vargaftik, B. N. Volkov, and L. D. Voljak, J. Phys. Chem. Ref. Data 12, 817 (1983). 